\title{
ABCB1 c.3435C >T POLYMORPHISM IS ASSOCIATED WITH PLATINUM TOXICITY: A PRELIMINARY STUDY
}

Beatrice De Troia ${ }^{1}$, Davide Dalu ${ }^{1}$, Virginio Filipazzi ${ }^{1}$, Luigi Isabella ${ }^{1}$, Nicoletta Tosca ${ }^{1}$, Sabrina Ferrario $^{1}$, Anna Rita Gambaro ${ }^{1}$, Luisa Somma ${ }^{1}$, Cinzia Fasola ${ }^{1}$, Stefania Cheli ${ }^{2}$, Emilio Clementi ${ }^{3,4}$, Davide De Francesco $^{5}$, Felicia Stefania Falvella ${ }^{2}$ and Maria Teresa Cattaneo ${ }^{1}$

${ }^{1}$ Department of Oncology, ASST Fatebenefratelli-Sacco, Milan, Italy

${ }^{2}$ Unit of Clinical Pharmacology. Department of Laboratory Medicine, ASST Fatebenefratelli-Sacco, Milan, Italy

${ }^{3}$ Scientific Institute, IRCCS E. Medea, Bosisio Parini, Lecco, Italy;

${ }^{4}$ Unit of Clinical Pharmacology, Department of Biomedical and Clinical Sciences, Consiglio Nazionale delle Ricerche Institute of Neuroscience, University Hospital "Luigi Sacco", Università di Milano, Milan, Italy

${ }^{5}$ Department of Infection and Population Health, UCL, London, UK

\section{Correspondence to:}

Beatrice De Troia

Department of Oncology, ASST Fatebenefratelli-Sacco, Milan, Italy, Via Giovanni Battista Grassi, 74 20157, Milan, Italy

e-mail contact: beadt83@gmail.com

Keywords: lung cancer, platinum, genetic factors, efflux transporters, toxicity 


\section{ABSTRACT}

Background: Platinum-based doublets are the standard chemotherapy for lung cancer. The identification of markers associated with drug-toxicity may improve the success of the treatment. Single nucleotide polymorphisms (SNPs) mapping into the genes involved in platinum transport or detoxification may explain occurrence of toxicities. In this study, we evaluated the role of 3 SNPs in predicting the onset of adverse events for lung cancer patients receiving cisplatin or carboplatin in adjuvant, neo-adjuvant and metastatic settings.

Methods: Eighty-two patients affected by non-small-cell and small-cell lung cancer treated with cisplatin- or carboplatin-based chemotherapy (stage II-IV) were enrolled. Before genetic analysis, patients signed a written informed consent. DNA was extracted from peripheral blood samples and genotypes were determined by Real-Time PCR. We selected and analyzed 3 SNPs: $A B C B 1$ c.3435C > T/rs 1045642, ABCC2 -24C>T/rs717620 and GSTP1 c.313A>G/rs1695. Patient characteristics and genotypes were correlated with haematological, gastrointestinal and renal toxicity as recorded by Common Terminology Criteria for Adverse Event (CTCAE) v4.03. No neurological toxicity was observed in our patients.

Results: Variant alleles were present in $53 \%$ of patients for $A B C B 1$ c.3435C>T, $18.3 \%$ for $A B C C 2$ 24C>T, and $34.8 \%$ for GSTP1 c.313A>G.

Heterozygous $\mathrm{CT}$ at $A B C B 1$ c.3435 were associated to a lower risk of haematological toxicity compared to homozygous $\mathrm{CC}(\mathrm{OR}=0.20 ; 95 \% \mathrm{CI} 0.05,0.69 ; \mathrm{p}=0.01)$. Similar results were observed by genetic dominant model (CT+TT vs CC) and haematological toxicity (OR=0.26; 95\% CI 0.09, 0.79; $\mathrm{p}=0.02)$. No other significant associations were found between toxicity and SNPs. Multivariate analysis confirmed an independent value for the $A B C B 1 \mathrm{c} .3435 \mathrm{C}>\mathrm{T}$ polymorphism.

Conclusions: The present study reveals that $A B C B 1$ c.3435C $>\mathrm{T}$ polymorphism influences platinum toxicity. The T allele seems to exert a protective effect on the development of toxicities. Further studies, such as epigenetic regulation ones, are needed to validate and shed more light on this association.

\section{INTRODUCTION}

Lung cancer, both of the small-cell and non small cell type, is one of the leading causes of cancer death in many countries. It is the second most common cancer in both men and women, not counting skin cancer [1].

The American Cancer Society's estimated 222,500 (116,990 in men and 105,510 in women) new cases of lung cancer in 2017 in the United States and about 155,870 deaths from it (84,590 in men and 71,280 
in women). Lung cancer mainly occurs in elderly. About 2 out of 3 people diagnosed with lung cancer are 65 or older, less than $2 \%$ are younger than 45 and the median age at diagnosis is about 70 years [2]. The risk is much higher for smokers than for non-smokers [3]. Statistics on survival vary depending on the stage at diagnosis [2].

Platinum-based chemotherapy is the first-line treatment for patients diagnosed at an advanced stage; however, drug resistance and toxicity may significantly undermine the successful treatment [4].

Cisplatin was the first member of this class, approved by FDA in 1978. Cisplatin acts as alkylating agent with the platinum-DNA complexes inhibiting DNA synthesis and transcription. Similar to other antineoplastic agents, cisplatin causes significant adverse drug reactions (ADRs) including nephrotoxicity, neurotoxicity and myelosuppression. Carboplatin was approved in 1989 and it has rarely been reported to be associated with nefrotoxicity. It is considered to be less neurotoxic than cisplatin while having a major risk of myelotoxicity [5].

Many factors, including genetic ones, explain the occurrence of the platinum-based chemotherapy adverse events. Understanding why some patients present severe toxicity while others do not, could improve the success of chemotherapy. Genes encoding for efflux transporters and/or drug detoxification have been investigated for the potential accumulation of platinum compounds into the cells. However, conflicting and inconclusive results leave open the question regarding their value in clinical practice. The ATP binding cassette $(\mathrm{ABC})$ superfamily of transportes, includes the main transporters responsible for efflux of platinum. Among them ABCB1 was the first to be evaluated in association with platinum efficacy and/or toxicity. The $A B C B 1$ gene encodes for the membrane-associated protein P-glycoprotein (P-gp) that acts as efflux pump with broad substrate specificity. The synonymous single nucleotide polymorphism (SNP) $A B C B 1$ c.3435C > T/rs 1045642 was the most widely investigated since, Hoffmeyer et al. showed association of the c.3435 TT genotype with low expression of P-gp in the gut and increased plasma levels of digoxin compared to the c.3435 CC genotype [6]. Subsequent replication studies have however produced inconsistent results as extensively reviewed by Leschzine et al. [7]. ABCC2, encoding for MRP2 protein, is another widely investigated platinum transporter. Recently, it has been reported that a 5'-UTR variant (-24C>T/rs717620) may predict platinum-based chemotherapy response [8,9]. Indeed, MRP2 has broad-substrate specificity for hydrophilic compounds, including the glutathione conjugates of platinum compounds. Glutathione-S-transferase P1 (GSTP1) is the most investigated GST enzyme, c.313A>G/rs1695 polymorphism induces Ile105Val substitution that reduced enzyme function by altering the interaction of catalytic domain and a variety of substrates [10]. 
In this study we evaluated the effect of these selected functional variants, which account for impaired cellular efflux and platinum detoxification, on the occurrence of several toxicities in lung cancer patients treated with platinum-based chemotherapy.

\section{PATIENTS AND METHODS}

\section{Patient population and treatment}

The study included 82 lung cancer patients recruited at the Oncology Department ASST Fatenenefratelli Sacco of Milan, between September 2014 and March 2017. Inclusion criteria included histologically confirmed diagnosis of non small-cell or small-cell lung cancer (I-IV), age $\geq 18$ years, ECOG performance status $\leq 2$, no previous antiblastic treatment and adequate renal, hepatic and bone marrow function. Patients with concomitant neoplastic disease other than lung cancer, psychiatric and infectious diseases were excluded from enrollment. Patients were treated with platinum-based chemotherapy. Cisplatin was used in association with gemcitabine, pemetrexed, docetaxel, vinorelbine etoposide or in monochemotherapy and carboplatin was associated with etoposide or vinorelbine. A blood sample for DNA extraction and analysis was taken at the beginning of the treatment. Demographic data, medical history and adverse drug reactions were collected. Dose reductions of chemotherapy, as well as treatment delays and permanent discontinuations were also recorded. All patients provided written informed consent prior to blood withdrawal and pharmacogenetics analysis. In each patient, toxicity was evaluated and graded by Common Terminology Criteria for Adverse Event (CTCAE) v4.03.

\section{Genotyping}

Genomic DNA was isolated from peripheral blood cells using an automatic DNA extraction system (Maxwell@ 16 System, Promega) according to the manufacturer instructions. DNA concentration and purity were evaluated by absorbance methodology using a NanoDrop 1000 Spectrophotometer V3.7 (Thermo Scientific). SNPs were determined by Real-Time PCR, using a panel of LightSNiP from TIBMolBiol (assays based on SimpleProbe $\left.{ }^{\circledR}\right)$. The SimpleProbe $®$ included in the LightSNP assay can detect single-base mismatches, thus enabling analysis of polymorphisms. At the end of the amplification a melting curve analysis is performed (LightCycler 480, Roche). We evaluated the functional variants mapping in $A B C B 1 \quad($ c.3435C $>\mathrm{T} / \mathrm{rs} 1045642), \quad A B C C 2 \quad(-24 \mathrm{C}>\mathrm{T} / \mathrm{rs} 717620)$ and GSTP1 (c.313A $>\mathrm{G} / \mathrm{rs} 1695)$ genes.

\section{Statistical analysis}


The primary outcome of the study was the development of haematological, gastrointestinal, and renal toxicity as recorded by Common Terminology Criteria for Adverse Event (CTCAE) v4.03. The HardyWeinberg equilibrium was evaluated using the $\chi^{2}$ test. Toxicity was defined as absent or mild when the severity grade was between 0 and 2 and as severe when the severity grade was 3 or 4 . Associations between platinum-toxicities and demographic and clinic-pathological characteristics, chemotherapy regimens and genotypes were assessed using the $\chi^{2}$ test, Fisher's exact test or Wilcoxon signed rank test, as appropriate. Odds ratios associated with selected SNPs were obtained using logistic regression with the wild type as reference category. In order to control for potential confounders, patient characteristics and genotypes associated with severe haematological toxicity in univariate analysis ( $p$-value $<0.1)$, entered in a multivariate logistic regression model to evaluate their independent association with severe toxicity. Analyses were performed using R version 3.2.4.

\section{RESULTS}

\section{Patient characteristics and toxicity outcomes}

Of the 82 enrolled patients, 52 (63.4\%) were males and 30 (36.6\%) were females. Seventy-six (92.7\%) patients were affected by non-small cell lung cancer and $6(7.3 \%)$ patients by small-cell lung cancer. All patients received platinum-based chemotherapy: 39 (47.6\%) patients received cisplatin in association with gemcitabine, $16(19.5 \%)$ cisplatin plus pemetrexed, 11 (13.4\%) cisplatin with docetaxel, $6(7.4 \%)$ cisplatin plus vinorelbine, 2 (2.4\%) cisplatin and etoposide, 2 (2.4\%) cisplatin in monotherapy, $5(6.1 \%)$ carboplatin with etoposide and only 1 patient (1.2\%) received carboplatin in association with vinorelbine. A total of $21(25.6 \%)$ patients developed severe haematological toxicity, $13(15.9 \%)$ and $2(2.4 \%)$ developed severe gastrointestinal and renal toxicity, respectively. None of the patients developed severe neurological toxicity. Treatment protocol modifications were necessary for $6(7.4 \%)$ patients and permanent discontinuation for $25(30.5 \%)$. No significant association with severe toxicity was observed for age, gender, smoking status, histological type, chemotherapy regimens, total drug dosage, drug dosage adjusted for body surface and cycles of chemotherapy. A marginally significant association was found between body surface area and severe haematological toxicity $(\mathrm{OR}=0.07 ; 95 \% \mathrm{CI} 0.01,1.36$; $\mathrm{p}=0.09)$.

\section{Genotyping results and association between SNPs and toxicity}

All selected functional SNPs were successfully genotyped in our cases and were found to be in HardyWeinberg equilibrium ( $\mathrm{p}>0.05)$. $A B C B 1$ c.3435C $>$ T was present in 39 (47.6\%) patients in heterozygosis and in $24(29.3 \%)$ in homozygosis, $A B C C 2-24 \mathrm{C}>\mathrm{T}$ in $20(24.4 \%)$ in heterozygosis and in $5(6.1 \%)$ in 
homozygosis, GSTP1 c.313A>G in 37 (45.1\%) in heterozygosis and in 10 (12.2\%) in homozygosis. For each polymorphism the variant alleles have been considered according to a dominant model (heterozygous + homozygous): the $A B C B 1$ c.3435 CT+TT genotypes were present in 63 (76.8\%), the ABCC2 -24 CT+TT in $25(30.5 \%)$ and the GSTP1 c.313 AG+GG in 47 (57.3\%) patients. Severe haematological toxicity resulted associated with $A B C B 1$ c. $3435 \mathrm{C}>\mathrm{T}$, in particular CT genotype appeared protective for the development of haematological toxicity $(\mathrm{OR}=0.20 ; 95 \%$ CI $0.05,0.69 ; \mathrm{p}=0.01)$ compared to CC genotype. Similar results were observed by genetic dominant model (CT+TT vs CC) for haematological toxicity $(\mathrm{OR}=0.26 ; 95 \% \mathrm{CI} 0.09,0.79 ; \mathrm{p}=0.02)$ as shown in Table 1 . No other significant association was found between SNPs and gastrointestinal and renal toxicity. Finally, the association between $A B C B 1$ c. $3435 \mathrm{C}>\mathrm{T}$ and severe haematological toxicity remained significant in multivariate analysis accounting for the body surface area (CT vs CC OR=0.18; 95\% CI 0.05, 0.65; $\mathrm{p}=0.01$ and $\mathrm{CT}+\mathrm{TT}$ vs CC OR=0.24; 95\% CI 0.07, 0.75; $\mathrm{p}=0.01$; Table 2).

\section{DISCUSSION}

Haematotoxicity is a major complication on many chemotherapy regimens. Although several factors are involved in this adverse event, genetic ones may play a relevant role. The identification and use of genetic predictors of platinum toxicity would be a good tool for the prevention of life-threatening side effect. In this study, we investigated whether polymorphisms in genes encoding for efflux transporters and/or drug detoxification $(A B C B 1, A B C C 2$ and $G S T P 1)$ were associated with toxicity to platinum-based chemotherapy in 82 lung cancer patients. Cellular transport proteins can influence drug absorption, distribution, and excretion and are key determinants of platinum accumulation into the cells and, potentially, for the development of the toxicity [11]. Haematological toxicities were the most detected toxicities (25.6\% of patients) followed by gastrointestinal (15.9\%) and renal (2.4\%) toxicities. Our result show that severe haematological toxicity is associated with the $A B C B 1$ c.3435C>T polymorphism. In particular the CT genotype appeared to exert a protective effect in the development of the toxicity compared to the CC genotype. The dominant genetic model (CT+TT) also revealed a similar significant association with haematological toxicity.

The product of $A B C B 1, \mathrm{P}$-gp, is expressed in the apical membrane of cells with excretory functions like liver, kidney, stomach, small intestine, and blood brain barriers, thus acting as a gate keeper against numerous xenobiotics $[12,13]$.

$A B C B 1$ expression is highest in haematopoietic progenitor cells CD34+ [14-15] and this could explain the role of $A B C B 1$ c.3435C $>$ T polymorphism in haematological toxicity. In our study the variant allele 
(CT+TT) seems to be associated with a 74\% lower risk of toxicity compared to CC genotype. The association between $A B C B 1$ c.3435 (CT+TT vs CC) and severe haematological toxicity remained also significant in multivariate analysis accounting for the body surface area. The association of this polymorphism and cisplatin-induced toxicity is controversial. Isla et coll. in a group of 62 lung cancer patients treated with cisplatin plus docetaxel observed a major risk of grade $\geq 2$ diarrhea in $33 \%$ of patients homozygous for the TT genotype of $A B C B 1$ c.3435 polymorphism, in contrast of those CT and CC [16]. Another study conducted on 671 patients treated with irinotecan and cisplatin or etoposide and cisplatin showed that the $A B C B 1$ c.3435 TT SNP was associated with almost a 4-fold increased risk of irinotecan-associated grade 3 or worse diarrhea as compared with CC and CT; combined grade $\geq 3$ neutropaenia and diarrhea was 5 times more likely in $A B C B 1$ c.3435 TT and UGT1A1*28 AA compared to $\mathrm{CC}$ and $\mathrm{CT}$ and GG and GA, respectively [17].

Other studies didn't find significant association between ABCB1 c.3435 C>T polymorphism and cisplatin toxicity $[18,19,20]$.

Different genetic association results from different studies, may be explained by several factors such as the limited sample size of the patients included in the studies, heterogeneity of treatment protocols, treatment intent (curative vs palliative) and ethnicity. Regarding ethnicity, the distribution of the $A B C B 1$ c.3435C $>\mathrm{T}$ polymorphism varies significantly between populations. In the present study allele $\mathrm{T}$ was present in 53\% of patients, a proportion similar to European (52\%) but different from American (43\%), Asian (40\%) and African (15\%) (www.ensembl.org). The involvement of $A B C B 1$ c.3435C>T polymorphism in the development of the toxicity is unclear. The $A B C B 1$ c.3435 $\mathrm{T}$ allele may be a functional allele or a marker of a functional allele that is regulated in tissue specific manner.

It is known that P-gp controls the passage of several drugs across cell membranes and, similar to our finding, a meta-analysis indicates that methotrexate toxicity was associated with the $A B C B 1$ c. $3435 \mathrm{C}>\mathrm{T}$ polymorphism in rheumatoid arthritis under an overdominant model (CT vs. CC + TT; OR 0.483, $95 \%$ CI $0.259-0.900, \mathrm{p}=0.022$ ), with a lower risk of methotrexate toxicity for heterozygotes CT compared to homozygotes (CC and TT) [21].

Because of its role, an alteration of P-gp expression or function may cause a difference in terms of efficacy and toxicity of chemotherapeutic agents $[22,23]$. Anyway cisplatin is not a substrate of Pglycoprotein and its efficacy and toxicity are not ensured by P-gp efflux activity, in fact several cell regulatory pathways may be altered in $\mathrm{P}$-gp positive cell, including protein expression, glycosylation and phosphorylation and may, consequentially, be involved in reduced cell sensitivity to substances that are not P-gp substrates [24,25,26]. 
Interestingly, as reported by Gibalová et al., differences in apoptosis induced by cisplatin were observed between P-gp-positive and P-gp-negative cells. More in details cisplatin induced a more marked entry in apoptosis in P-gp-negative cells than in positive ones. This response acts as a secondary cellular response independently of P-gp efflux activity; altering cell sensitivity to drugs other than those that are substrates of P-gp. [27].

Similarly X.-Y. Liu et al. demonstrate that expression of MDR1 gene, the gene that encodes the drug efflux protein P-gp, is higher in the the HCC cell line HepG2, resistant to cisplatin. Moreover, this acquired resistance is associated with hyperactivation of the c-Jun N-terminal kinases (JNK), an important anti-apoptosis pathways, whereas JNK inhibition increases sensitivity to cisplatin. These results underscore a close association between resistance to cisplatin in HCC cells, MDR1 overexpression, and the JNK signaling pathway [28] .

In conclusion, the present study reveals that $\mathrm{ABCB} 1$ c. $3435 \mathrm{C}>\mathrm{T}$ polymorphism may influence platinum toxicity. Variant $\mathrm{T}$ allele could exert a protective effect on the development of toxicities. Further studies with a larger sample size and/or epigenetic regulation studies are warranted to validate these findings

\section{COMPLIANCE WITH ETHICAL STANDARDS}

\section{Conflict of Interests}

All the Authors declare they have no conflict of interest.

\section{Ethical approval}

All procedures performed in studies involving human participants were in accordance with the ethical standards of the institutional and/or national research committee and with the 1964 Helsinki declaration and its later amendments or comparable ethical standards.

\section{Informed consent}

Informed consent was obtained from all individual participants included in the study.

\section{REFERENCES}

1. Siegel RL, Miller KD, Jemal A. Cancer Statistics, 2017. CA Cancer J Clin. 2017; 67 (1):7-30. doi: 10.3322/caac. 21387 .

2. American Cancer Society (2017). Cancer Facts \& Figures 2017. Atlanta; American Cancer Society. 
3. Cornfield J, Haenszel W, Hammond EC, Lilienfeld AM, Shimkin MB, Wynder EL (2009). Smoking and lung cancer: recent evidence and a discussion of some questions. Int J Epidemiol 38 (5): 1175 1991. doi: 10.1093/ije/dyp289.

4. S. Novello, F. Barlesi, R. Califano, T. Cufer, S. Ekman, M. Giaj Levra, K. Kerr, S. Popat, M. Reck, S. Senan, G. V. Simo, J. Vansteenkiste, S. Peters (2016). Metastatic non-small-cell lung cancer: ESMO Clinical Practice Guidelines for diagnosis, treatment and follow-up. Ann Oncol 27 (suppl_5): v1-v27. doi: 10.1093/annonc/mdw326.

5. Ho GY, Woodward N, Coward JI (2016). Cisplatin versus carboplatin: comparative review of therapeutic management in solid malignancies. Rev Oncol Hematol Jun 102:37-46. doi: 10.1016/j.critrevonc.2016.03.014.

6. Hoffmeyer S, Burk O, von Richter O, Arnold HP, Brockmöller J, Johne A, Cascorbi I, Gerloff T, Roots I, Eichelbaum M, Brinkmann U (2000). Functional polymorphisms of the human multidrugresistance gene: multiple sequence variations and correlation of one allele with P-glycoprotein expression and activity in vivo. Proc Natl Acad Sci USA Mar 28 97(7):3473-8. doi: 10.1073/pnas.050585397

7. Leschziner GD, Andrew T, Pirmohamed M, Johnson MR (2006). ABCB1 genotype and PGP expression, function and therapeutic drug response: a critical review and recommendations for future research. Pharmacogenomics J Jun 7(3):154-79. doi: 10.1038/sj.tpj.6500413.

8. Sun N, Sun X, Chen B, Cheng H, Feng J, Cheng L, Lu Z (2009). MRP2 and GSTP1 polymorphisms and chemotherapy response in advanced non-small cell lung cancer. Cancer Chemother Pharmacol. 2010 Feb 65(3):437-46. doi: 10.1007/s00280-009-1046-1.

9. Han ZG, Tao J, Yu TT, Shan L (2017). Effect of GSTP1 and ABCC2 Polymorphisms on Treatment Response in Patients with Advanced Non-Small Cell Lung Cancer Undergoing Platinum-Based Chemotherapy: A Study in a Chinese Uygur Population. Med Sci Monit Apr 26 23:1999-2006. doi: 10.12659/MSM.904156. 
10. Ali-Osman F, Akande O, Antoun G, Mao JX, Buolamwini J (1997). Molecular cloning, characterization, and expression in Escherichia coli of full-length cDNAs of three human glutathione Stransferase Pi gene variants. Evidence for differential catalytic activity of the encoded proteins. J Biol Chem Apr 11 272(15):10004-12. doi: 10.1074/jbc.272.15.10004.

11. Evans WE, McLeod HL (2003) Pharmacogenomics — drug disposition, drug targets, and side effects. N Engl J Med 348:538-549. doi: 10.1056/NEJMra020526.

12. Lum BL, Gosland MP (1995) MDR expression in normal tissues. Pharmacologic implications for the clinical use of P-glycoprotein inhibitors. Hematol Oncol Clin North Am 9:319-336. doi: 10.1177/00912709922011755.

13. Milojkovic M, Stojnev S, Jovanovic I, Ljubisavljevic S, Stefanovic V, Sunder-Plassman R. (2011) Frequency of the C1236T, G2677T/A and C3435T MDR1 gene polymorphisms in the Serbian population. Pharmacol Rep PR 63:808-814. doi: 10.1016/S1734-1140(11)70593-X.

14. Bunting KD (2002). ABC transporters as phenotypic markers and functional regulators of stem cells. Stem Cells 20:274. doi: 10.1634/stemcells.20-3-274.

15. Chaudhary PM, Roninson IB (1991). Expression and activity of P-glycoprotein, a multidrug efflux pump, in human hematopoietic cells. Cell 66:85. doi: http://dx.doi.org/10.1016/0092-8674(91)90141-K.

16. Isla D, Sarries C, Rosell R, Alonso G, Domine M, Taron M, Lopez-Vivanco G, Camps C, Botia M, Nuñez L, Sanchez-Ronco M, Sanchez JJ, Lopez-Brea M, Barneto I, Paredes A, Medina B, Artal A, Lianes P (2004). Single nucleotide polymorphisms and outcome in docetaxel-cisplatin treated advanced non-small cell lung cancer. Annals of Oncology 15:1194-1203. doi: 10.1093/annonc/mdh319.

17. Primo N. Lara Jr, Ronald Natale, John Crowley, Heinz Josef Lenz, Mary W. Redman, Jane E. Carleton, James Jett, Corey J. Langer, J. Philip Kuebler, Shaker R. Dakhil, Kari Chansky, and David R. Gandara (2009). Phase III trial of irinotecan/cisplatin compared with etoposide/cisplatin in extensivestage small cell lung cancer: clinical and pharmacogenomic results from SWOG S0124. J Clin Oncol. 27: 2530-2535. doi: 10.1200/JCO.2008.20.1061. 
18. Qian C-Y, Zheng Y, Wang Y, Chen J, Liu JY, Zhou HH, Yin JY, Liu ZQ. Associations of genetic polymorphisms of the transporters organic cation transporter 2 (OCT2), multidrug and toxin extrusion 1 (MATE1), and ATP-binding cassette subfamily $\mathrm{C}$ member 2 (ABCC2) with platinum-based chemotherapy response and toxicity in non-small cell lung cancer patients. Chin J Cancer 2016; 35:85. doi: 10.1186/s40880-016-0145-8.

19. Zair ZM and Singer DR. Efflux transporter variants as predictors of drug toxicity in lung cancer patients: systematic review and meta-analysis. Pharmacogenomics 2016; 17:1089-112. doi: 10.2217/pgs2015-0006.

20. Chen S, Huo X, Lin Y, Ban H, Lin Y, Li W, Zhang B, Au WW, Xu X. Association of MDR1 and ERCC1 polymorphisms with response and toxicity to cisplatin-based chemotherapy in non-small-cell lung cancer patients. Int J Hyg Environ Health 2010 Mar;213(2):140-5. doi: 10.1016/j.ijheh.2010.01.004

21. Lee YH, Bae SC, Song GG (2016). Association of the ABCB1 C3435T polymorphism with responsiveness to and toxicity of DMARDs in rheumatoid arthritis: A meta-analysis. Z Rheumatol Sep 75(7):707-15. doi:10.1007/s00393-015-1618-x.

22. Pallis, M., Russell, N. Strategies for overcoming p-glycoprotein-mediated drug resistance in acute myeloblastic leukaemia. Leukemia 2004; 18 (12), 1927-1930. doi: 10.1038/sj.leu.2403511.

23. Pallis, M., Turzanski, J., Higashi, Y., Russell, N. P-glycoprotein in acute myeloid leukaemia: therapeutic implications of its association with both a multidrugresistant and an apoptosis-resistant phenotype. Leuk. Lymphoma 2002; 43 (6), 1221-1228. doi: $10.1080 / 10428190290026277$.

24. Hamaguchi, K., Godwin, A.K., Yakushiji, M., O’Dwyer, P.J., Ozols, R.F., Hamilton, T.C. Crossresistance to diverse drugs is associated with primary cisplatin resistance in ovarian cancer cell lines. Cancer Res. 1993; 53 (21), 5225-5232. 
25. Yang, X., Page, M. P-glycoprotein expression in ovarian cancer cell line following treatment with cisplatin. Oncol. Res. 1995; 7 (12), 619-624.

26. Demeule, M., Brossard, M., Beliveau, R.. Cisplatin induces renal expression of P-glycoprotein and canalicular multispecific organic anion transporter. Am. J. Physiol. 1999; 277 (6 Pt 2), F832-F840. doi: 10.1152/ajprenal.1999.277.6.F832.

27. Gibalová L, Sereš M, Rusnák A, Ditte P, Labudová M, Uhrík B, Pastorek J, Sedlák J, Breier A, Sulová Z. P-glycoprotein depresses cisplatin sensitivity in L1210 cells by inhibiting cisplatin-induced caspase-3 activation. Toxicol In Vitro 2012; 26:435-444. doi: 10.1016/j.tiv.2012.01.014.

28. X.-Y. Liu, S.-P. Liu, J. Jiang, X. Zhang, T. Zhang. Inhibition of the JNK signaling pathway increases sensitivity of hepatocellular carcinoma cells to cisplatin by down-regulating expression of Pglycoprotein. Eur Rev Med Pharmacol Sci. 2016;20(6):1098-108. 